\title{
Osteogenesis imperfecta type III in South Africa: Psychosocial challenges
}

\author{
L X G Stephen, ${ }^{1,2}$ BChD, PhD; T Roberts, ${ }^{1-3}$ BChD, MChD, PhD candidate; E van Hayden; M Chetty, ${ }^{1-3}$ BChD, MChD, PhD candidate \\ ${ }^{1}$ Faculty of Dentistry, University of the Western Cape, Cape Town, South Africa \\ ${ }^{2}$ University of Cape Town/University of the Western Cape Dental Genetic Clinic, Red Cross War Memorial Children's Hospital, Cape Town, \\ South Africa \\ ${ }^{3}$ Division of Human Genetics, Department of Pathology, University of Cape Town, South Africa
}

Corresponding author: $M$ Chetty (drmchetty@mweb.co.za)

\begin{abstract}
Individuals with osteogenesis imperfecta type III (OI III) are severely physically disabled due to frequent fracturing. Their disability poses numerous barriers that challenge their social development. Despite these limitations, several affected persons are able to rise above these problems and achieve success in their personal and professional life. This outcome is directly relevant to their psychosocial development. The achievements of five individuals with OI III living in Cape Town are highlighted in this article, as well as the challenges that they have experienced and continue to experience in their daily lives. The authors intend to promulgate understanding of the psychosocial circumstances of affected persons, thereby facilitating the deployment of appropriate efforts and resources to address these challenges.

S Afr Med J 2016;106(6):S90-S93. DOI:10.7196/SAMJ.2016.v106i6.11005
\end{abstract}

Osteogenesis imperfecta (OI) is a heterogeneous group of inherited connective tissue disorders characterised by skeletal fragility, frequent fracturing and variable bone deformity. The primary pathological defect is an abnormality in type I collagen and, in addition to the skeleton, other tissues such as the teeth, tendons, sclerae and skin may also be affected.

The most widely used classification of OI is that proposed by Sillence et al.$^{[1]}$ describing four main types. This classification is based upon an analysis of clinical and genetic factors (Table 1).

OI type III (OI III) is an uncommon yet well-defined autosomal recessive condition in which marked impairment of growth, bone fragility and severe progressive deformity are major characteristics. At birth, affected individuals present with multiple fractures and limb deformities. The fractures continue into childhood and long bones tend to bend, resulting in a disturbance of growth and, often, consequent stunted stature. The neck and trunk are relatively short and a discrepancy in the ratio of arm span to height is consistently found. Kyphoscoliosis is a frequent presentation and is age-related, with the majority of adults having severe spinal deformity. Death usually occurs during the first and second decades of life due to complications arising from the severe bone fragility, marked skeletal deformity, pulmonary hypertension and cardiopulmonary failure. ${ }^{[1]}$ The condition is well recognised in South Africa (SA). ${ }^{[2]}$

Radiographic features are a porotic skeleton as well as the consequences of several fractures. The long bones are usually bowed and Wormian bones are evident in the cranium. Vertebral biconcavity, protusio acetabulae and elongation of the pedicles of the lumbar vertebrae are important diagnostic criteria. ${ }^{[2]}$

Since 2006, a growing list of mutant genes causing the $5-10 \%$ of recessive cases of OI has emerged. These include CRTAP, LEPRE1, PPIB, PLOD2, SERPINH1, SERPINF1, BMP1, TMEM38B, SP7, WNT1, CREB3L1 and FKBP10. These genes are responsible for the multistep process that involves a number of post-translational modifications in the processing, assembly and transportation of procollagen chains during the biosynthesis of collagen type I.

OI III does not compromise intellectual ability and some individuals with the condition succeed in school and the workplace.
They are able to develop and maintain satisfying social and family relationships and acquire a remarkable sense of independence. Affected individuals invariably face many challenges such as limited mobility, physical pain, lack of access to medical and social services, social prejudices and architectural access barriers. ${ }^{[3]}$

\section{Objectives}

The objective of this article is to highlight the psychosocial challenges and lifelong barriers encountered by a group of individuals diagnosed with OI III in SA and to document their successful efforts in gaining full participation in society.

It is through recording their experiences that barriers in their lives could be identified. Another objective of the project was to improve the understanding of the psychosocial circumstances of affected individuals, thereby facilitating appropriate efforts and resources to address these challenges.

\section{Methods}

The authors became acquainted with five individuals with a confirmed diagnosis of OI III who were convened by EvH in her leadership capacity within her community in Cape Town. They were of Cape mixed ancestry heritage with no known relationship to each other, and were of a similar socioeconomic standing in their community. These individuals ranged in age from 12 to 61 years and despite their significant physical disability, they had been

Table 1. Classification of OI

\begin{tabular}{lll}
\hline Type & Clinical features & $\begin{array}{l}\text { Genetic factors: } \\
\text { Mode of inheritance }\end{array}$ \\
\hline I & Blue sclera, moderate bone fragility & Autosomal dominant \\
II & Lethal in the perinatal period & Autosomal recessive \\
III & $\begin{array}{l}\text { White sclera, severe with } \\
\text { progressive deformity }\end{array}$ & Autosomal recessive \\
IV & White sclera, variable bone fragility & Autosomal dominant
\end{tabular}


Table 2. Age, general physical condition, social and academic achievements of the study participants

\begin{tabular}{lllll}
\hline Participant & Age (years) & General physical condition & Education & Current job description \\
\hline 1 & 61 & Chairbound & Grade 10 & Retired bookkeeper, jewellery maker \\
2 & 28 & Calipers & Grade 12, degree in graphic design & Graphic designer, dancer \\
3 & 28 & Calipers & Grade 12, BA(Hon) in journalism & Journalist, public speaker \\
4 & 22 & Chairbound & Grade 12, diploma in computer programming & Beauty therapist, enrolled for a BA degree \\
5 & 12 & Chairbound & Grade 5 & Scholar, swims as a hobby
\end{tabular}

relatively successful in terms of occupational, academic and social achievements. These study participants and their unaffected parents completed questionnaires designed to identify their psychosocial challenges and the efforts they had made in overcoming these disadvantages. This article records the information acquired from these discussions and the questionnaire.

\section{Results}

OI III, a severe autosomal recessive form of OI, has been studied in SA for more than 40 years. ${ }^{[2]}$ One of the participants in this survey, EvH (participant 1), and her family had been documented in 1975 in the early medical genetic literature when she was 22 years old. ${ }^{[4]}$ This individual had taken the initiative to reach out to similarly affected persons in her community in Cape Town and in this context she played a major role in this project (Table 2). To demonstrate the manifestations of the disorder, $\mathrm{EvH}$, at her own request, is depicted at 22 and 61 years (Fig. 1 and Fig. 2, respectively). She wrote the following subjective view of the impact of the condition on her own life:

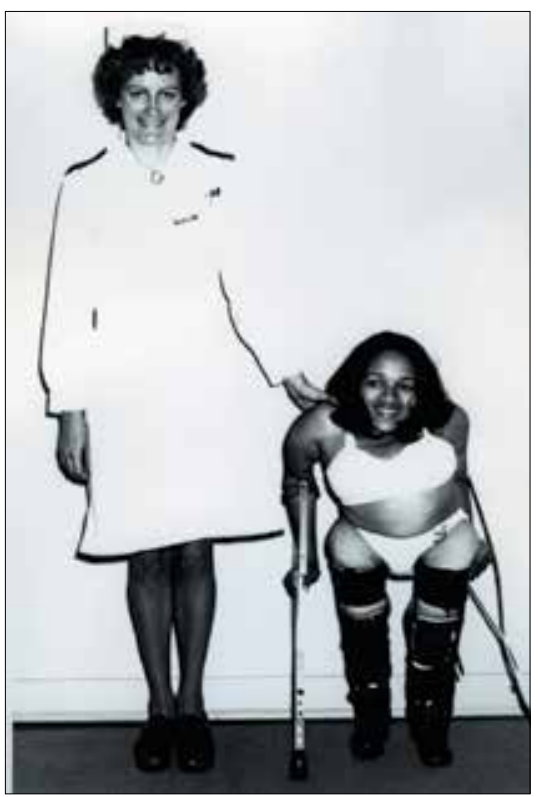

Fig. 1. EvH at 22 years. A young woman in callipers. (Picture courtesy of Prof. P Beighton. ${ }^{[4]}$ )
I am the sixth child in a family of seventeen siblings from the Eastern Cape. Three other siblings were also affected, but passed on in early childhood. I walked for the first time at the age of 8 years with the use of bilateral callipers and ring crutches. Although I was elated with my new found independence, it was also the time that I encountered the fear of falling - a reality that I constantly live with, having seriously fractured my bones more than 50 times over the years.

At the age of 3 years, my parents placed me at St Joseph's Home, an institute for disabled children in Cape Town. At age 14 years, I was placed in the care of relatives in Durban because they were the only family that was willing to take me in. For the next 8 years, I was maid, cook, nanny and on the receiving end of their constant emotional abuse.

That was a turning point in my life when I finally accepted my disability and limitations and made a conscious decision to be successful in all aspects of my life. In 1973, at 22 years old, with the help of a social worker, I travelled to Cape Town to attend an industrial training centre where I acquired various vocational skills, was subsequently able to earn a living and for the

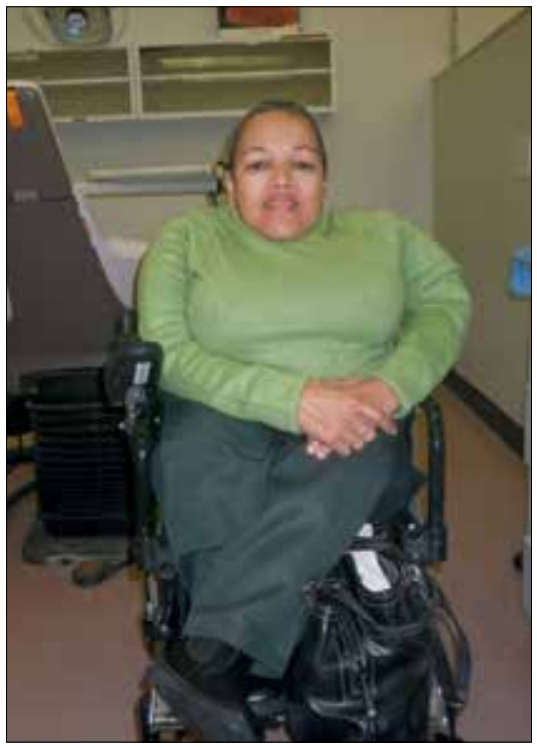

Fig. 2. EvH at 61 years is chairbound. first time in my life, I was free. I travelled, attended the theatre and went to jazz clubs. Being only $97 \mathrm{~cm}$ in height, the greatest challenge was travelling in public transport and it was during these times that I stumbled upon humiliating and embarrassing encounters from people, which included obscenities hurled at me and an attempted rape. While being rescued from one of these encounters, I met my future husband. We have been married for 25 years and I am able to have a normal marriage in every sense of the word.

Over the years, I learnt that I had to be friendly, engaging and vocal about my needs in order to be assisted in an environment that was not conducive to my disability. In return for all the help I received in every aspect of my life, my goal is to be of service to others similarly affected, share my experiences and hopefully enrich their lives.

Analysis and discussion of feedback Parental reflections

The parents of participants 2, 3, 4 and 5 indicated that they felt an initial sense of shock, anxiety and depression at the realisation that they had a child with marked physical disability. These feelings subsequently gave rise to helplessness and loneliness, compounded by anger and the fear of the unknown. All parents reported a serious compromise in their financial situation and stated that it was a matter of several years before they regained a sense of balance in this regard. Of the five families, the parents of only one individual embarked upon a further pregnancy after the birth of the affected child.

The parents began to understand more about their offspring's disability with the help of medical professionals and began participating in the healthcare needs of the child. It is relevant that the parents of three of the affected individuals indicated that accessing information regarding their child's condition 25 - 30 years ago was difficult and almost impossible.

All the parents emphasised the need for emotional support and accessible counselling 
services, which would have helped allay their fears and concerns. They also highlighted that they actively sought out parents of similarly affected children in order to discuss and develop coping mechanisms.

Empowered with knowledge of the condition, the parents realised that the main responsibility in a chronic illness is care. Most parents were closely involved in all aspects of their young child's life, notably mobility, grooming and the clinical therapeutic management. Each participant experienced numerous fractures, which ranged from 30 to 50 in number, and had to undergo many medical tests, treatments and surgeries; these circumstances made it difficult for them to live a 'normal' life. Parents of these young children had to come to terms with the fact that despite their careful handling, they could inadvertently cause their child to fracture.

As toddlers, four of these individuals were immobilised in plaster several times due to their fracturing. Hospital visits were a frequent reality and their parents had to ensure that these children felt safe and secure. However, due to lack of facilities, it was impossible for these children to receive enough stimulation to compensate for their inability to move around. Parents reported that their children often fell behind scholastically due to prolonged hospitalisation. They highlighted the importance of the inclusion of an educational programme while receiving treatment.

Due to their lack of access to adequately equipped schools for physically disabled children, these children were placed, by their parents, in mainstream schools. It was at this stage of the children's development that parents found it difficult to control their children's need for physical movement. When these individuals reached adolescence, although parents felt the need to relinquish their role as sole providers, the lack of options and community resources available to these adolescents resulted in the parents continued pivotal role in their children's lives in order to facilitate the smooth transition into adulthood. The parents of participants 2, 3 and 4 eventually realised that their overprotectiveness lessened the likelihood of their children developing autonomy, selfesteem and motivation. Eventually, despite trepidation, they resigned themselves to a less interactive role.

\section{Affected individuals}

At school as children, the affected individuals encountered physical barriers such as high desks, lack of wheelchair ramps, no adapted toilet facilities and absence of safe playground facilities. They also had to endure and eventually learnt to cope with stares and comments from other children. The advantage of going to school was the fact that they made friends. These friends helped them establish their social life. Activities such as music, drama and swimming elicited favourable responses from the affected children.

Overprotection, fear and anxiety were identified as unintentional attitudes that placed limitations on participation in activities and added to the students' feeling of isolation and difference. General lack of knowledge of the medical and psychosocial aspects of individuals with OI III was reported as a continuous social barrier.

To the best of the authors' knowledge, no information has been published in the SA literature concerning the intellectual capabilities of OI III-affected persons and the psychosocial barriers that these individuals encounter. It is highly relevant that, despite severe disability and economic constraints, individuals 2, 3 and 4 have acquired graduate degrees from the University of Cape Town and have pursued careers in dance, drama and business.

The authors' interaction with these persons confirmed their engaging nature. All of them demonstrated advanced social skills and it was this trait that enabled them to communicate their condition to unfamiliar persons such as physicians and therapists, hence taking increased responsibility for their own care.

The five affected persons have achieved complete independence for all basic personal tasks. Adaptive devices such as reachers, bedpans and modified clothing, as well as innovative designs in the bathroom and kitchen, were key to their independence. To compensate for their short arms, four individuals make use of a laptop computer and a voice-activated typing system. Driving was another important aspect in their transition to independence. Four individuals have drivers' licences and drive modified vehicles.

The participants reported that maintaining an appropriate body weight for their height was a constant challenge. Although they are aware of the need for good nutrition, active movement and strengthening exercises, they indicated a scarcity of resources and guidance in this regard.

All five affected individuals, together with their parents, expressed concern when medical records were transferred from the paediatric to the adult care facility. They reported a lack of knowledge about OI III and the services which were available from the adult caregivers. All individuals reported difficulty in obtaining a family doctor's services and in accessing a physician's office. The adult patients expressed regret that their disability had at one time or another prevented them from receiving appropriate health care. Four individuals were denied dental treatment by dentists who admitted that they were not adequately trained and that their clinical environment was not conducive to treating persons with this condition.

These affected persons have learned important ways to prevent fractures and continue to lead an active and fulfilled life. They pay attention to body mechanics and safe exercise; in particular correct lifting techniques, good posture and strength training are all employed.

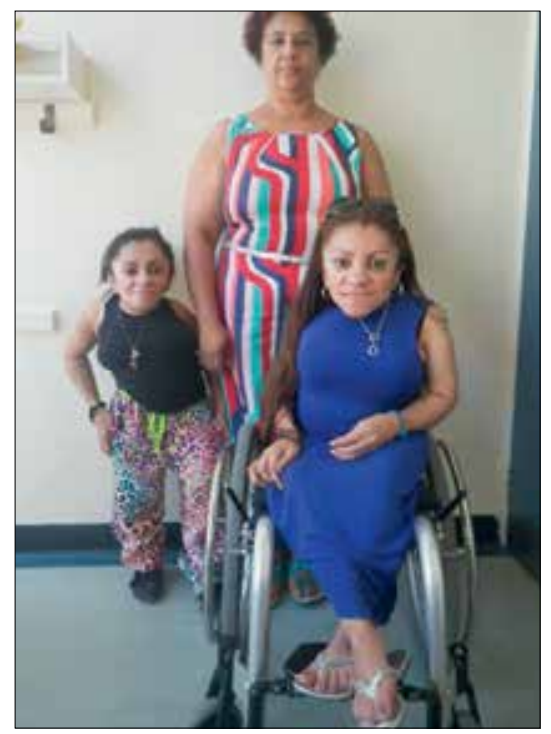

Fig. 3. Participants 2 and 3. Twin sisters aged 28 years with their mother.

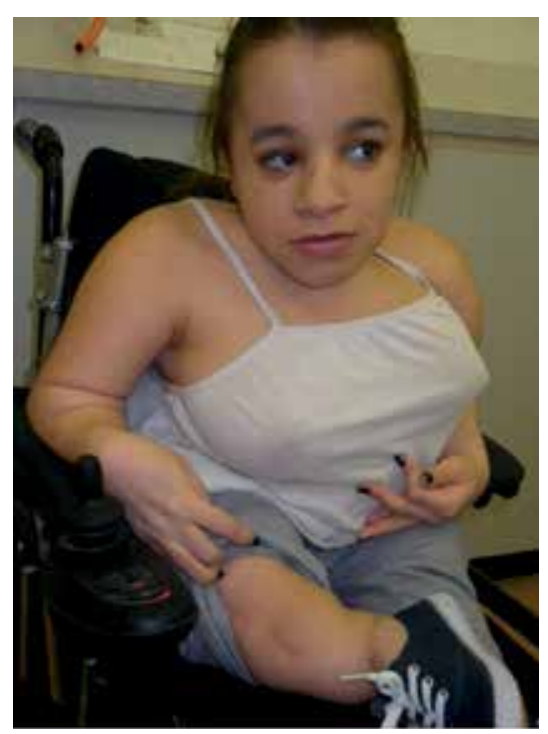

Fig. 4. Participant 4 aged 22 years. She is chairbound and has marked physical disability and short stature. 
All affected persons expressed a keen interest in personal grooming, fashion trends, romantic relationships and concern with regard to the functional and aesthetic condition of their dentition (Figs 3 and 4).

\section{Conclusion}

Children in SA have the right to be educated in a state school and to receive equitable quality education that prepares them academically and socially for life outside the classroom. An observation noted in all five individuals, which is in keeping with previous statements by other authors, is that persons with OI III differed from their non-disabled peers only in terms of their relative hypoactivity and motor performance, due to their severe disability. ${ }^{[5]}$

For children with limited mobility, physical barriers may prevent their full participation in school activities. The most common barriers noted include steps at school entrances or between floors, restrooms with narrow doors, high sinks, narrow corridors which are inaccessible by wheelchair, far-off playgrounds, work areas that are difficult to reach for persons of short stature or who are chairbound. According to official education regulations, these barriers must be addressed if they interfere with a child receiving his or her education. Affected individuals must have access to safe and reliable transport which must involve wheelchair access. In reality, the information from statements of affected persons and their parents suggests that these regulations are not applied.
Since the hallmark of OI III is frequent fracturing, it is important that teachers, friends and work colleagues learn how to deal with a fracture in a calm and appropriate manner. Often the affected person is able to instruct others in the most appropriate way to respond to his or her fracture.

The individuals documented in this report have shown remarkable social and academic success despite a lack of resources in terms of family counselling and social support, as well as ill equipped schools, and lack of assistance in the transition from childhood to adolescence and finally to independence in adulthood.

An interdisciplinary treatment approach for individuals with OI III would prepare parents of children with physical challenges to accept and help their adolescents' transition into the adult world. Improved support in the psychosocial aspects of care faced by the parents is necessary. Ideally, an interdisciplinary team should cover genetic counselling and social support for parents, and the medical, dental, orthopaedic, anaesthetic, nursing care, rehabilitation and nutrition needs of these individuals, from childhood into adulthood.

\section{References}

1. Sillence DO, Senn A, Danks DM. Genetic heterogeneity in osteogenesis imperfecta. J Med Genet 1979;16(2):101-116. DOI:10.1136/mg.16.2.101

2. Beighton P, Spranger J, Versfeld G. Skeletal complications in OI: Review of 153 South African patients. S Afr Med J 1983;64(15):565-568.

3. Suskauer SJ, Cintas HL, Marini JC, Gerber LH. Temperament and physical performance in children with osteogenesis imperfecta. Pediatrics 2003;111(2):E153-E161. DOI:10.1542/peds.111.2.e153

4. Horan F, Beighton P. Autosomal recessive inheritance of osteogenesis imperfecta. Clin Ge 1975;8(2):107. DOI:10.1111/j.1399-0004.1975.tb04398.x

5. Widmann RF, Laplaza FJ, Bitan FD, Brooks CE, Root L. Quality of life in osteogenesis imperfecta. In Orthop 2002;26(1):3-6. DOI:10.1007/s002640100292 